\title{
A New EIFS Strategy for Performance Improvement of IEEE 802.11 Wireless LANs
}

\author{
Ho-Ting $\mathrm{Wu}$ \\ Department of Computer Science and Information Engineering, National Taipei \\ University of Technology, 1, Sec. 3, Chung-Hsiao E. Rd., Taipei, Taiwan, R.O.C.
}

Tel: 886-2-27712171 ext. 4221 E-mail: htwu@csie.ntut.edu.tw

\begin{abstract}
Chia-Wei Tuan (Corresponding author)
Department of Computer Science and Information Engineering, National Taipei University of Technology, 1, Sec. 3, Chung-Hsiao E. Rd., Taipei, Taiwan, R.O.C.

E-mail: chiawei74105@yahoo.com.tw
\end{abstract}

\begin{abstract}
Mei-Ting Chuang
Department of Computer Science and Information Engineering, National Taipei University of Technology, 1, Sec. 3, Chung-Hsiao E. Rd., Taipei, Taiwan, R.O.C.

E-mail: ufvkomj@hotmail.com
\end{abstract}

\begin{abstract}
In this paper, we propose a new EIFS strategy to improve the performance in IEEE 802.11. In IEEE 802.11 specifications, the EIFS time delay is activated after a node initiates an unsuccessful transmission. The new EIFS strategy uses the same activation mechanism as the specifications. The major difference between the standard EIFS and the new EIFS strategy is when to deactivate such time delay. According to the specification, a node deactivates the EIFS time delay whenever any node in system executes a successful transmission. However, in our proposed EIFS strategy, a node deactivates the EIFS time delay only when it has completed a successful transmission. Performance results via simulations have shown that this new strategy is able to boost the system performance significantly.
\end{abstract}

Keywords: WLAN, IEEE 802.11, DCF, EIFS. 


\section{Introduction}

With the increasing number of portable devices, wireless local area network (WLAN) plays an important role in communication industry. IEEE 802.11 groups define the physical layer mechanisms such as the signal synchronization, data scrambler, convolution encoder, interleaving, and modulation $[1,2]$. However, in this paper, we will not discuss the design issues in physical layer.

The nodes in WLAN share the same transmission medium and must follow a predefined medium access rules. The medium access control (MAC) mechanisms are specified in [3]. Two functions defined in IEEE 802.11 MAC layer are optional PCF (Point Coordinated Function) and mandatory DCF (Distributed Channel Function). PCF is employed in contention-free period (CFP) while DCF is employed in contention period (CP). A CP and a $\mathrm{CFP}$ are contained in a periodic superframe depicted in Figure 1.

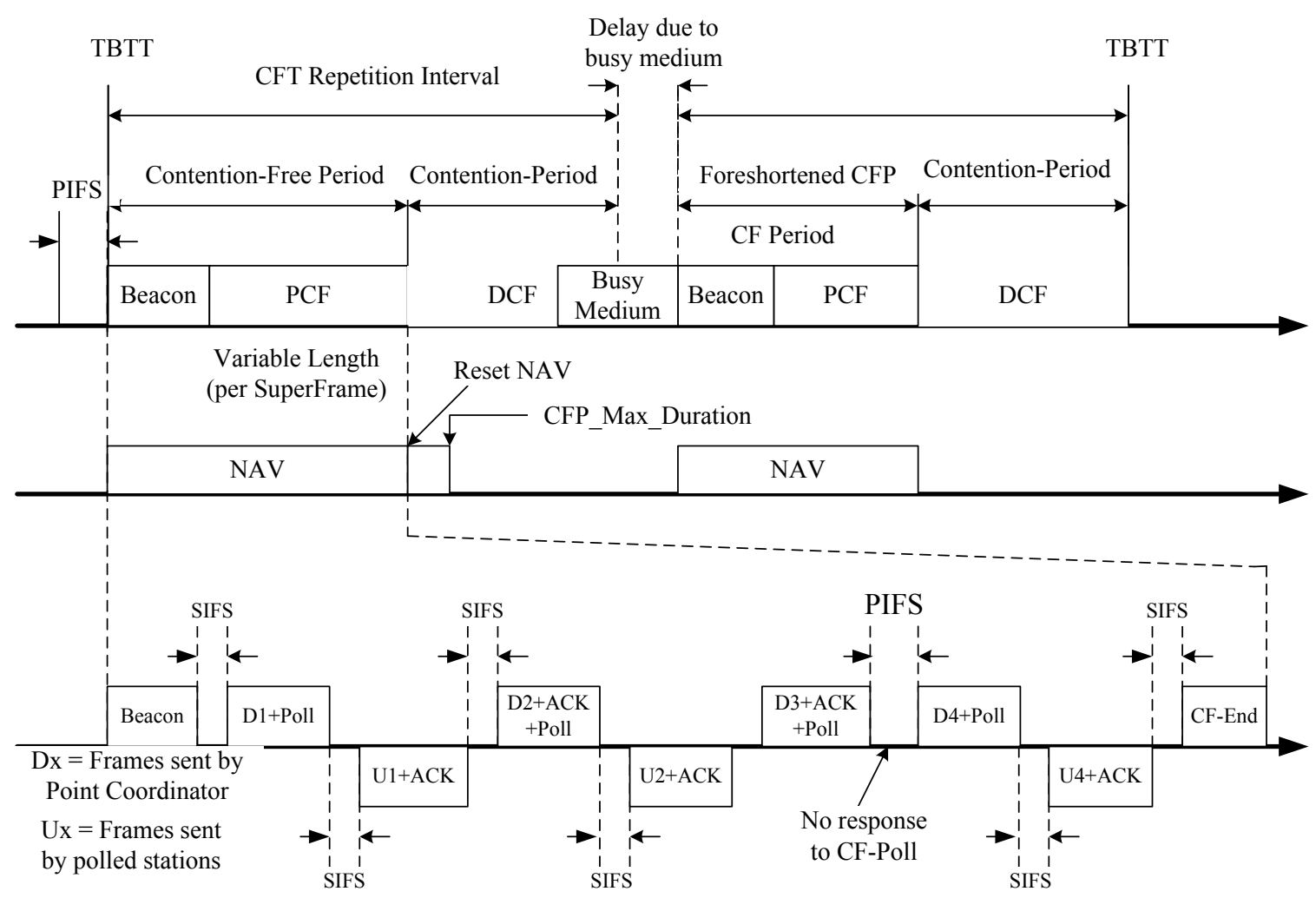

Figure 1. The coexistence of CFP and CP.

PCF is a centralized polling scheme. Access point (AP) polls each station for its turn to transmit data. No stations are allowed to transmit unless it is polled. PCF is seldom, if ever, implemented in IEEE 802.11 products. Therefore, we only focus on DCF in this paper.

DCF is a contention-oriented scheme based on Carrier Sense Multiple Access with Collision Avoidance (CSMA/CA). CSMA/CA is adopted due to the property of WLAN where it is impossible to listen while sending data, thus a random length of delay is necessary before access the medium. Figure 2 shows the access mechanism in DCF. In a normal situation, once a station wishes to send a data frame, it should defer for a DCF Inter-Frame 
Space (DIFS) and then backoff a random delay time based on Contention Window (CW). The backoff time is decreased slot by slot when the channel is idle. The transmission starts when the backoff time reaches zero. If two or more stations choose the same value of backoff time, collision of frames happen. The value of $\mathrm{CW}$ is determined as follows. Initially, $C W$ is set as $C W_{\text {min }}$. Whenever the transmitter detects that there is an error in the transmitted frame (e.g., collision or physical noise), it sets $C W_{\text {new }}=2 \times\left(C W_{\text {old }}+1\right)-1$. However, the value of $C W$ must conform to the inequality, $C W_{\min } \leq \mathrm{CW} \leq C W_{\max }$. Finally, backoff time is determined by BackoffTime $=$ Random $[0, \mathrm{CW}] \times$ aSlotTime, where the function Random $[x, y]$ returns a random integer value between $x$ and $y$ inclusively and aSlotTime depends on the physical parameters defined in the specifications. After receiving an error-free transmission, the receiver should delay one Short Inter-Frame Space (SIFS) then transmit an acknowledgment (ACK) back to the sender. It is noted that the PCF Inter-Frame Space (PIFS) is used for PCF mechanism, which is not discussed in the paper.

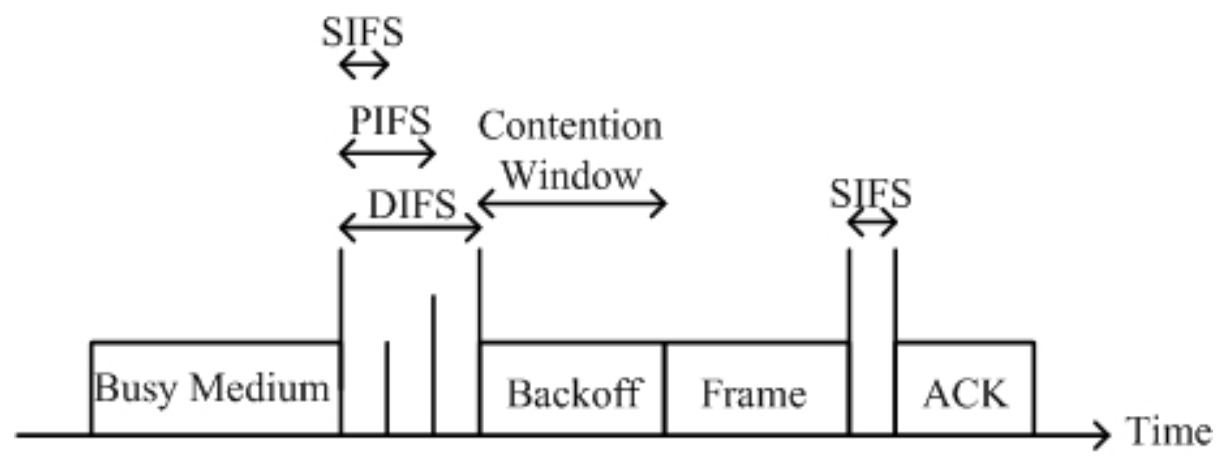

Figure 2. Access mechanism in DCF.

The longest inter-frame space is Extended Inter-Frame Space (EIFS). The station defers for EIFS instead of DIFS once the station receives an erroneous frame. In this paper, we will propose a new EIFS strategy for the performance improvement of IEEE 802.11.

The rest of this paper is organized as follows. In the next section, we will discuss the previous works for the performance improvement of IEEE 802.11 wireless LANs. In Section 3, the original EIFS strategy defined in the specifications and proposed EIFS strategy will be described. In Section 4, the illustrative performance results in terms of normalized throughput, blocking probability, and mean delay via simulations are given. Finally, conclusions are drawn in Section 5.

\section{Related Works for Performance Improvement of IEEE 802.11 wireless LANs}

The demand of higher throughput in IEEE 802.11 motivates researchers to design adequate algorithms to improve system performance. The simplest way to support higher throughput is supporting higher data rate in physical layer. Indeed, IEEE 802.11a supports higher data rate than IEEE 802.11b. However, [4] shows that a theoretical throughput limit exists, even if the data rate is infinite. The throughput is bounded by the limit due to MAC layer and PHY layer overheads. Therefore, pursuing higher data rates is not enough. Reducing the overhead is also important. 
In order to improve the performance of IEEE 802.11 effectively, there are lots of works on call admission control mechanisms and adaptive contention window adjustment algorithms.

A call admission control mechanism is used to prevent the insufficient bandwidth situation in the system. Before a new flow joins the system, the transmitter sends a traffic specification to AP. The AP must ensure that the new joining flow would not affect the requirement of the existing stations in system. If there is not enough bandwidth, AP will reject the new flow, otherwise it accepts this new flow. The call admission control mechanism proposed in [5]_works well in terms of system performance. However, call admission controls rely on infrastructured IEEE 802.11 WLANs. That is, it does not work in a distributed system such as ad hoc network.

The adaptive contention window adjustment algorithms are used to adjust the CW dynamically. With a larger number of stations, the CW must be large enough to reduce the collision probability. This class of algorithms often invokes complicated mathematics such as queueing analysis. However, due to the complexity of the network behavior, many researchers such as $[6,7]$ assume that the network is under saturation condition. That is, there is at least one frame in the buffer at any station. Obviously, it is not the scenario in the real network.

In this paper, we do not use these typical methods to improve the system performance. Instead, we propose a new EIFS strategy which works well without the need of AP and the assumption of saturation traffic.

\section{The Proposed EIFS Strategy for IEEE 802.11}

According to IEEE 802.11 specifications, before a station starts the transmission, it should defer for a DIFS or an EIFS delay time and then starts a random CW counter for backoff. Once the backoff counter decreases to zero, the station starts its transmission.

The choice between DIFS and EIFS depends on the event of the last transmission. If the last event is an unsuccessful transmission (e.g., a collision), the station waits for an EIFS, otherwise it waits for a DIFS. We call such station which waits for an EIFS a retry station. But, at what time will a retry station become a non-retry station? According to the original mechanism in IEEE 802.11, a retry station becomes a non-retry station when one of the stations in the system has conducted a successful transmission.

The mechanism makes a retry station become a non-retry station in a short period of time. Thus, the simulation results in [8] show that such EIFS mechanism makes few impacts on performance aspect. That is the reason why there are only very few papers focusing on the impacts of the EIFS period. Actually, even a few network simulators do not implement the EIFS period due to the little effect on performance.

In this paper, we propose a new EIFS strategy. In the proposed EIFS strategy, a retry station would not become a non-retry station until it has completed a successful transmission event. The retry station has the responsibility to change its own state to a non-retry station. 


\section{Macrothink}

Network Protocols and Algorithms

ISSN 1943-3581

2009, Vol. 1, No. 1

Even if the other stations in the system have successful transmissions, the retry station does not change its state. In other words, our proposed EIFS strategy assumes the same EIFS activation mechanism as the specifications. The major difference between the standard EIFS and the new EIFS strategy is when to deactivate such EIFS time delay. In specifications, a station deactivates the EIFS time delay whenever any single station in system has conducted a successful transmission. However, in our proposed EIFS strategy, a node deactivates the EIFS time delay only when it has a successful transmission.

In the following section, we compare the performance between original EIFS mechanism and our proposed EIFS mechanism. The simulation results show that our proposal is able to achieve higher throughput, lower packet delay, and lower packet blocking probability than the original IEEE 802.11's.

\section{The Analysis of the Proposed EIFS Mechanism}

\subsection{Assumption, Definition, and Simulation Configuration}

\subsubsection{Assumptions}

In the following simulation, we assume the communication range in each station covers all stations in the IEEE 802.11 environment. In other words, there is no hidden station problem, and no exposed station problem. In addition, if there is no collision in transmissions, the receiver always receives error-free frames.

\subsubsection{Definitions}

The performance in IEEE 802.11 such as normalized throughput, blocking probability, and mean delay are defined in (1), (2), and (3), respectively.

$$
\text { Normalized throughput }=\frac{\text { total successful transmission time }}{\text { total simulation time }}
$$

$$
\text { Blocking probability }=\frac{\# \text { of frames blocked by buffer }}{\# \text { of total generated frames }}
$$

$$
\text { Mean delay }=\sum_{\substack{\text { successful } \\
\text { transmission } \\
\text { frames }}} \begin{gathered}
\text { (the moment of successful transmission }) \\
\text { \# of frames transmitted successfully }
\end{gathered}
$$

\subsubsection{Simulation Configurations}

The physical layer is IEEE 802.11 b. Each station has 50 frames in the buffer at most. The inter-arrival time between two packets are 0.2 second (i.e. constant bit rate). The data frame generated by each station contains 1500 Bytes. The values of CWmin and CWmax are set as 
31 and 1023, respectively.

\subsection{The Performance Comparisons Between The Proposed EIFS Mechanism and the Original EIFS Mechanism Defined in IEEE 802.11 Specifications.}

In the following, we simulate the two mechanisms in IEEE 802.11 and compare the difference between the two mechanisms. The comparisons of normalized throughput, blocking probability, and mean delay are drawn in Figure 3 to Figure 5, respectively.

In Figure 3, when the number of stations is more than 15, the normalized throughput with proposed EIFS mechanism is higher than the one which is with original EIFS mechanism by 0.1 . The explanations are given as follows.

First of all, we divide all stations into two groups. One group is for stations in retry state. Another group is for stations in non-retry state. The retry stations which defer for a long EIFS period have much less chance to access the channel. So, the non-retry stations which defer for a short DIFS period can contend for the channel as if there are not so many stations in the system. Therefore, the non-retry stations contend for the channel access chance again and again until they have no frame to transmit. On the contrary, in the original EIFS mechanism defined in IEEE 802.11 specifications, the retry stations change their state to non-retry state whenever any station in the system has a successful transmission, so the retry stations stay in the retry group for a very short period of time. Thus, the intensive contention still exists in the next contention round. Such intensive contention induces the higher collision probability and lower throughput.

Figure 4 shows the blocking probability of proposed mechanism is less than the original mechanism's. Intuitively, we may think the proposed EIFS mechanism would lead to a higher blocking probability since the retry stations could not start their transmission for a long period of time. It may runs out of the buffer spaces. However, from the other viewpoint, the non-retry stations do not have to wait for a long period of time to transmit. On the contrary, all stations in original EIFS mechanism may have to wait for much more time to start transmission since a higher probability of collision induces more retransmission times.

Figure 5 shows the mean delay of proposed mechanism is less than the original mechanism's. The stations in proposed EIFS mechanism have less retry counter value since the non-retry stations could contend for the channel as if there are not so many stations in the system. Thus, the non-retry stations could start transmission as soon as possible. 


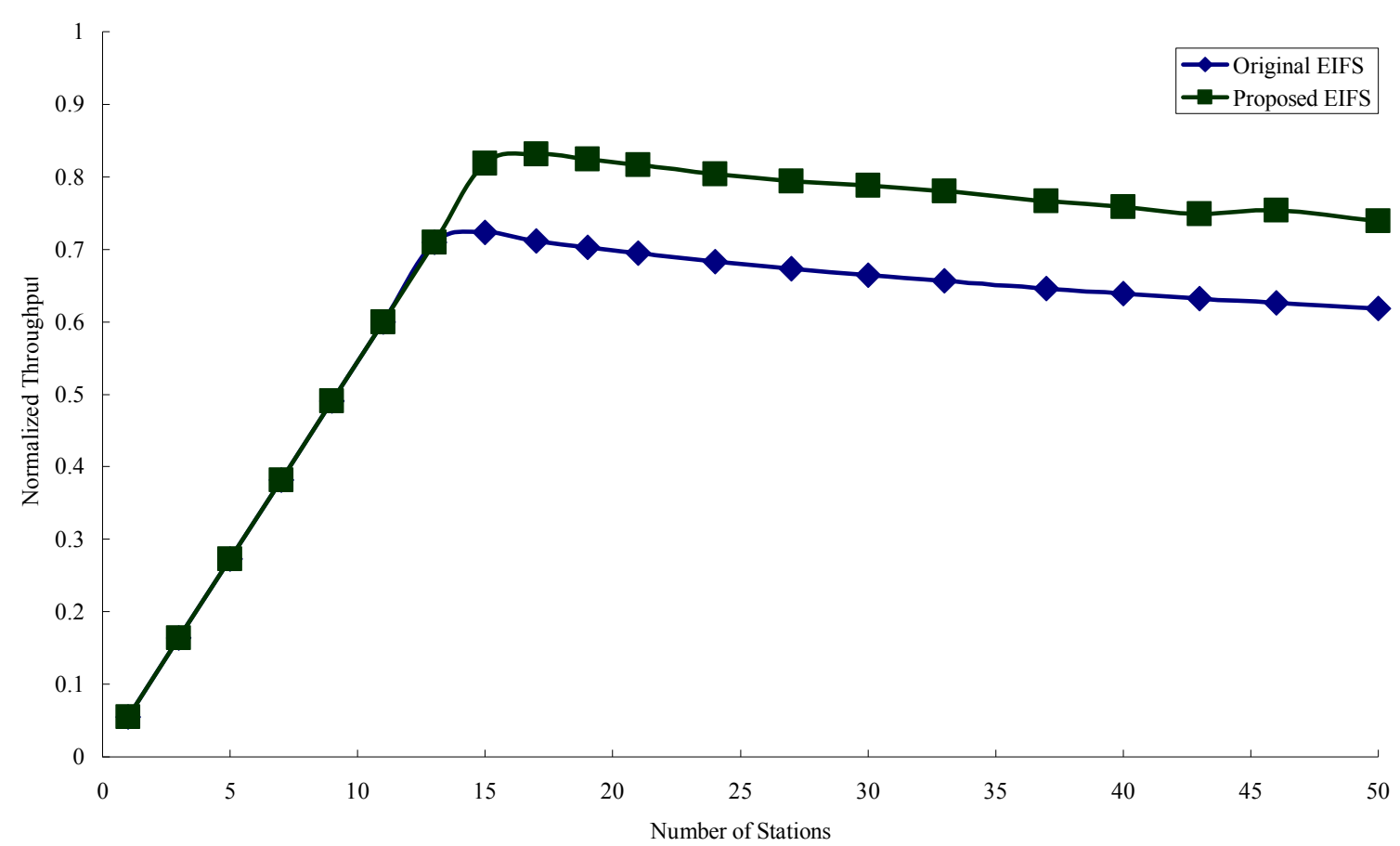

Figure 3. Comparison of normalized throughput between original EIFS and proposed EIFS strategy.

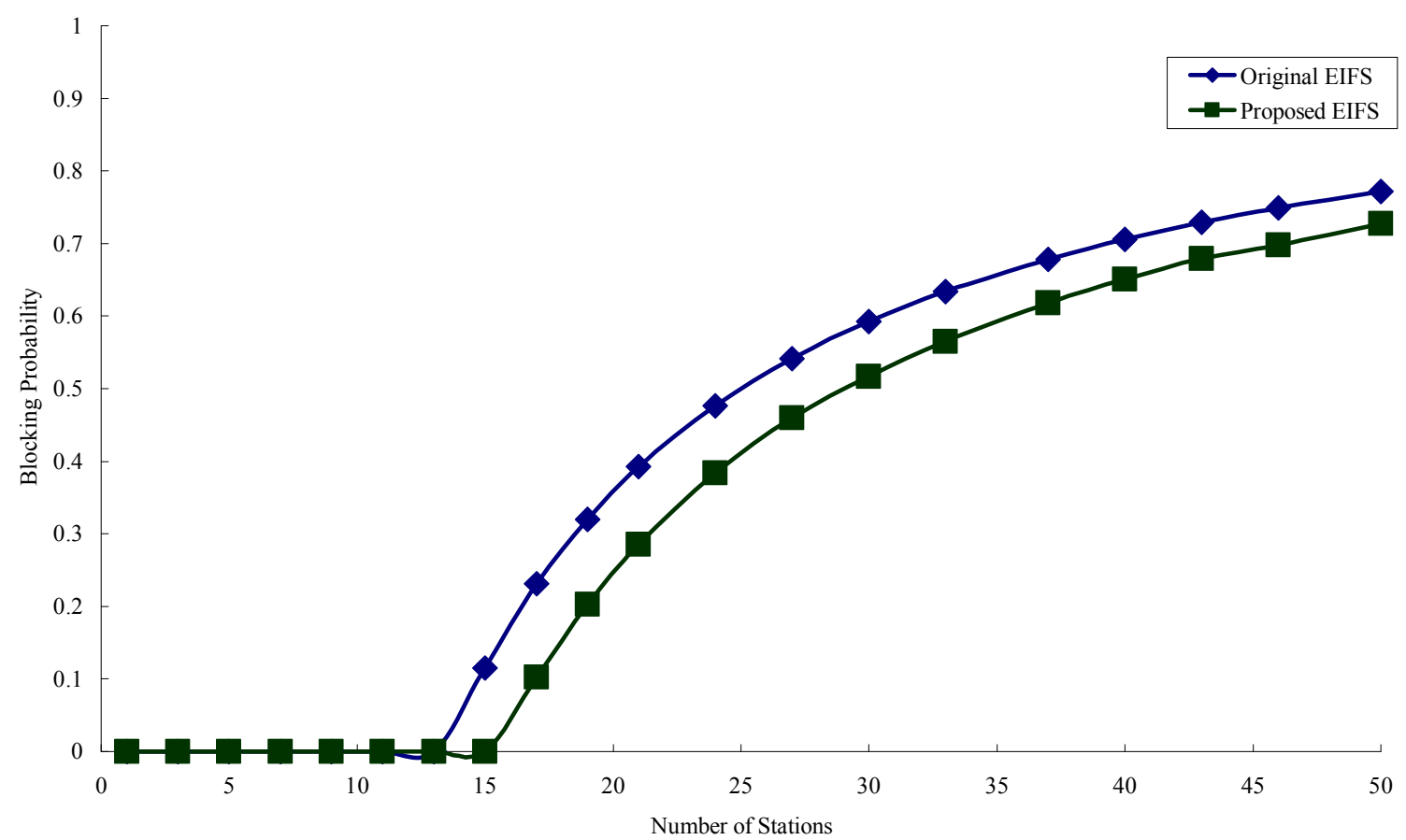

Figure 4. Comparison of blocking probability between original EIFS and proposed EIFS strategy. 


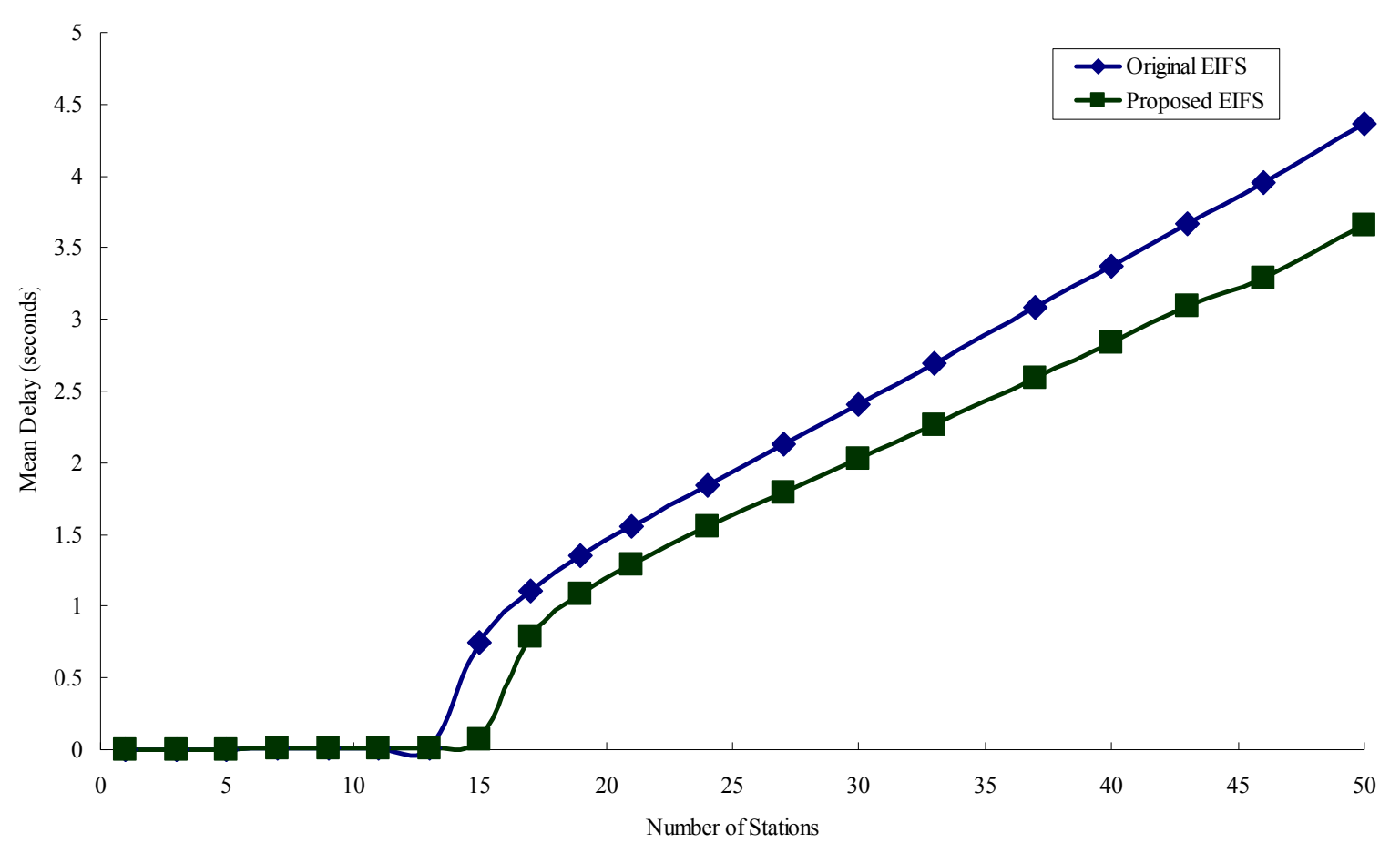

Figure 5. Comparison of mean delay between original EIFS and proposed EIFS strategy.

\section{Conclusions}

There are lots of researchers proposing diverse adaptive contention window adjustment algorithms and call admission control mechanisms to improve the system performance in IEEE 802.11. However, very few discussions on EIFS period can be found. In this paper, we instead propose a new EIFS strategy for the performance improvement. The simulation results show that the proposed EIFS mechanism works well in terms of throughput, blocking probability, and packet mean delay.

\section{Acknowledgement}

The authors would like to thank the anonymous reviewers for their constructive remarks and suggestions. Their comments enhanced the quality of this paper significantly.

\section{References}

[1] IEEE 802.11a WG. (2003). Supplement to IEEE Standard for Information technology-Telecommunications and information exchange between systems-Local and metropolitan area networks-specifications requirements Part 11: Wireless LAN Medium Access Control (MAC) and Physical Layer (PHY) specifications High-speed Physical Layer in the $5 \mathrm{GHz}$ Band.

[2] IEEE 802.11b WG. (2001). IEEE Standard for Information technology-Telecommunications and information exchange between systems Local and metropolitan area networks- Specifications requirements Part 11: Wireless LAN Medium Access Control (MAC) and Physical Layer (PHY) specifications Amendment 2: Higher-speed Physical Layer (PHY) extension in the 2.4 GHz band-Corrigendum 1. 


\section{Macrothink}

[3] IEEE 802.11 WG. (2007). IEEE Standard for Information technology-Telecommunications and information exchange between systems-Local and metropolitan area networks-Specific requirements - Part 11: Wireless LAN Medium Access Control (MAC) and Physical Layer (PHY) Specifications. (Revision of IEEE Std 802.11-1999).

[4] Yang Xiao. (2005). IEEE 802.11n: Enhancements for higher throughput in wireless LANs. IEEE Wireless Communications, 12(6), 82-91.

[5] Zhai, H., Chen, X., \& Fang, Y. (2006). A call admission and rate control scheme for multimedia support over IEEE 802.11 wireless LANs. Wirel. Netw. 12(4) (Jul. 2006), 451-463.

[6] Chetoui, Y., \& Bouabdallah, N. (2007). Adjustment mechanism for the IEEE 802.11 contention window: An efficient bandwidth sharing scheme. Computer Communications, 30 (13):2686-2695.

[7] El Housseini, S. \& Alnuweiri, H. (2005). Adaptive contention-window MAC algorithms for QoS-enabled wireless LANs. 2005 International Conference on Wireless Networks, Communications and Mobile Computing, vol.1 (13-16 June 2005), 368-374.

[8] Heindl, A. \& German, R. (2000). The impact of backoff, EIFS, and beacons on the performance of IEEE 802.11 wireless LANs. IEEE International on Computer Performance and Dependability Symposium, 103-112. 